\title{
21.
}

\section{Recherches sur les coefficients des facultés analytiques.}

(Par Mr. le Dr. O.Schlömilch, Prof. d'Analyse à l'école royale polytechnique de Dresde.)

En adoptant la définition de Mr. Crelle, nous appelerons faculté analytique une fonction de deux variables $z$ et $\mu$, qui satisfait aux deux conditions

$$
\text { (1.) } \quad f(z, \mu+k)=f(z, \mu) \cdot f(z+\mu, k)
$$

(2.) $f(z, 1)=z$.

De ces équations on tire, en faisant $k=1$ et $\mu=1,2,3$, etc.:

$$
\begin{aligned}
& f(z, 2)=f(z, 1) \cdot f(z+1,1)=z(z+1), \\
& f(z, 3)=f(z, 2) \cdot f(z+2,1)=z(z+1)(z+2), \\
& \text { etc., }
\end{aligned}
$$

et en désignant par $n$ un nombre entier positif:

(3.) $f(z, n)=z(z+1)(z+2) \ldots(z+n-1)$.

Pour trouver la valeur de $f(z, 0)$, nous faisons $\mu=0$ et $k=1$, ce qui donne

$$
f(z, 1)=f(z, 0) \cdot f(z, 1)
$$

et

$$
\text { (4.) } f(z, 0)=1 \text {. }
$$

Pour trouver la valeur de $f(z,-n)$, nous faisons $\mu=-n$ et $k=n$; l'équation (1.) donne alors

$$
f(\mathfrak{z}, 0)=f(z,-n) \cdot f(z-n, n),
$$

et par suite:

$$
f(z,-n)=\frac{f(z, 0)}{f(z-n, n)}=\frac{1}{(z-n)(z-n+1)(z-n+2) \ldots(z-n+n-1)},
$$

ou ce qui revient au même:

$$
\text { (5.) } \quad f(z,-n)=\frac{1}{(z-1)(z-2)(z-3) \ldots(z-n)}^{\circ}
$$

Nous nous bornerons à considérer les deux fonctions $f(z, n)$ et $f(z,-n)$, et nous les développerons en séries ordonnées suivant les puissances de la variable $z$. 
Quant à $f(z, n)$, il est clair, que l'on obtient une série finie de la forme

$$
\text { (6.) } \begin{aligned}
& f(z, n)=z(z+1)(z+2) \ldots(z+n-1) \\
= & \stackrel{n}{C_{0}} z^{n}+\stackrel{n}{C}_{1} z^{n-1}+\stackrel{n}{C}_{2} z^{n-2}+\cdots+\stackrel{n}{C}_{n-1} z,
\end{aligned}
$$

dans laquelle les symboles $\stackrel{n}{\boldsymbol{C}}_{0}, \stackrel{n}{\boldsymbol{C}}_{1}$, etc. désignent des coefficients encore inconnus que nous appelerons les coefficients des facultés à exposant positif. Il existe un développement semblable de la fonction $f(z,-n)$ que l'on peut mettre sous la forme

$$
f(z,-n)=\frac{\frac{1}{z}}{1-\frac{1}{z}} \cdot \frac{\frac{1}{z}}{1-\frac{2}{z}} \cdot \frac{\frac{1}{z}}{1-\frac{3}{z}} \cdots \frac{\frac{1}{z}}{1-\frac{n}{z}}
$$

et l'on obtient, en supposant $\boldsymbol{z}>\boldsymbol{n}$, la série infinie

$$
\text { (7.) } \begin{aligned}
& f(z,-n)=\frac{1}{(z-1)(z-2)(z-3) \cdots(z-n)} \\
= & \bar{C}_{0}^{n} z^{-n}+\bar{C}_{1}^{n} z^{-n-1}+\bar{C}_{2}^{n} z^{-n-2}+\bar{C}_{3}^{n} z^{-n-3}+\cdots ;
\end{aligned}
$$

ce qui est tout à fait analogue à l'équation (6.).

Si on n'a pour but que de construire une table des coefficients $C$ des deux équations (6. et 7.), on peut se servir des relations qui lient entre eux les coefficients de la même espèce. On obtient ces relations en ayant égard aux équations

$$
\begin{aligned}
f(z, n+1) & =(z+n) \cdot f(z, n) \text { et } \\
(z-(n+1)) \cdot f(z,-(n+1)) & =f(z,-n) .
\end{aligned}
$$

En substituant des séries équivalentes, ces équations se réduisent aux suivantes:

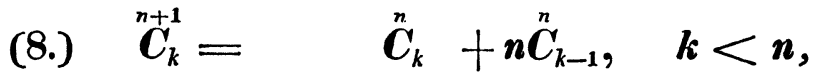

$$
\begin{aligned}
& \text { (9.) } \stackrel{-(n+1)}{\boldsymbol{C}_{k}}=(\boldsymbol{n}+1) \stackrel{-(n+1)}{\boldsymbol{C}_{k-1}}+\overline{\boldsymbol{C}}_{k}, \quad \boldsymbol{k} \geqq \boldsymbol{n} \text {. }
\end{aligned}
$$

Ces relations suffisent au calcul numérique, mais elles ne donnent pas une expression directe des coefficients cherchés. Elles offrent deux équations aux différences, dont il faut développer les intégrales finies. Pour y parvenir, on peut opter entre deux méthodes différentes. Le procédé le plus naturel serait d'exécuter l'intégration finie des équations ( 8 et 9.), mais on verra bientôt que cela exige des calculs assez longs, et qu'il est difficile de trouver la forme la plus convenable pour exprimer les coefficients cherchés. Le second procédé est, de prendre en secours le théorème de Mac-Laurin; ce qui donne 
immédiatement

$$
\text { (10.) } \quad \stackrel{n}{C}_{k}=\frac{1}{1.2 .3 \ldots(n-k)}\left[D^{n-k} f(z, n)\right]_{(z=())} .
$$

En faisant $z=\frac{1}{x}$ dans l'équation (7.), on a

(11.) $\frac{1}{(1-x)(1-2 x)(1-3 x) \cdots(1-n x)}=\bar{C}_{0}^{-n}+\bar{C}_{1}^{-n} x+\bar{C}_{2}^{-n} x^{2}+\bar{C}_{3}^{-n} x^{3}+\cdots$, et en désignant par $\varphi(x, n)$ l'expression à gauche:

$$
\text { (12.) } \quad \overline{\boldsymbol{C}}_{k}^{n}=\frac{1}{1.2 .3 \ldots k}\left[D^{k} \varphi(x, n)\right]_{(x=())} \text {. }
$$

À la première vue il paraît être difficile d'exécuter les différentiations indiquées des équations (10 et 12.), mais nous ferons voir que cette difficulté peut être évitée en fésant quelques transformations pour ramener les différentiations ci-dessus à la différentiation de quelques autres fonctions très simples. On parvient par là aux formules suivantes:

$$
\begin{aligned}
\ddot{C}_{k}^{n}= & \frac{(n)_{0} n^{n+k}-(n)_{1}(n-1)^{n+k}+(n)_{2}(n-2)^{n+k}-\cdots}{1.2 .3 \ldots n}, \\
\stackrel{+n}{\boldsymbol{C}_{k}=} & (n-1)_{k}\left[\frac{(n+k)_{0}(n+k-1)_{k}}{(2 k)_{k}} \bar{C}_{k}^{k}-\frac{(n+k)_{1}(n+k-2)_{k-1}}{(2 k-1)_{k-1}} \stackrel{(k-1)}{C}_{k}\right. \\
& \left.\quad+\frac{(n+k)_{2}(n+k-3)_{k-2}}{(2 k-2)_{k-2}} \stackrel{-(k-2)}{C}_{k}-\cdots\right] .
\end{aligned}
$$

Dans ces formules les symboles $(\mu)_{0},(\mu)_{1},(\mu)_{2}$, etc. désignent les coefficients du binôme, de sorte que

$$
(\mu)_{0}=1, \quad(\mu)_{1}=\frac{\mu}{1}, \quad(\mu)_{2}=\frac{\mu(\mu-1)}{1.2}, \text { etc. }
$$

Les formules (13 et 14.) étant établies, nous en donnerons quelques applications à la théorie des suites.

\section{\$. 1.}

Du coefficient $\overline{\boldsymbol{C}}_{k}^{n}$.

En désignant par $S_{n}$ la somme de la série finie

$$
\text { (1.) } \quad \frac{(n)_{0}}{a}-\frac{(n)_{1}}{a+1}+\frac{(n)_{2}}{a+2}-\frac{(n)_{3}}{a+3}+\cdots, 1
$$

on a, en multipliant par $\left(1+\frac{a}{n}\right)$ :

$$
\begin{aligned}
\left(1+\frac{a}{n}\right) S_{n}= & \frac{(n)_{0}}{a}-\frac{(n)_{1}}{a+1}+\frac{(n)_{2}}{a+2}-\frac{(n)_{3}}{a+3}+\cdots \\
& +\frac{(n)_{0}}{n}-\frac{(n)_{1}}{n} \frac{a}{a+1}+\frac{(n)_{2}}{n} \frac{a}{a+2}-\frac{(n)_{3}}{n} \frac{a}{a+3}+\cdots
\end{aligned}
$$


La seconde série peut être mise sous la forme

$$
\begin{gathered}
\frac{(n)_{0}}{n}-\frac{(n)_{1}}{n}\left(1-\frac{1}{a+1}\right)+\frac{(n)_{2}}{n}\left(1-\frac{2}{a+2}\right)-\cdots \\
=\frac{(n)_{0}-(n)_{1}+(n)_{2}-(n)_{3}+\cdots}{n}+\frac{1(n)_{1}}{n} \frac{1}{a+1}-\frac{2(n)_{2}}{n} \frac{1}{a+2}+\frac{3(n)_{3}}{n} \frac{1}{a+3}-\cdots \\
=\frac{(n-1)_{0}}{a+1}-\frac{(n-1)_{1}}{a+2}+\frac{(n-1)_{2}}{a+3}-\cdots
\end{gathered}
$$

parceque $(n)_{0}-(n)_{1}+(n)_{2}-\cdots=0$ et $\frac{k(n)_{k}}{n}=(n-1)_{k}$. L'équation donne maintenant

$$
\frac{n}{a+n} \boldsymbol{S}_{n}=\frac{(n)_{0}}{a}-\frac{(n)_{1}-(n-1)_{0}}{a+1}+\frac{(n)_{2}-(n-1)_{1}}{a+2}-\cdots,
$$

et comme on a toujours $(n)_{0}=1=(n-1)_{0}$ et

$$
(n)_{k}-(n-1)_{k-1}=(n-1)_{k},
$$

l'équation précédente se transforme en.

c'est à dire en

$$
\frac{n}{a+n} \boldsymbol{S}_{n}=\frac{(n-1)_{0}}{a}-\frac{(n-1)_{1}}{a+1}+\frac{(n-1)_{2}}{a+2}-\cdots
$$

$$
\frac{n}{a+n} \mathbf{S}_{n}=\mathbf{S}_{n-1} \quad \text { ou } \quad \boldsymbol{S}_{n}=\frac{n}{a+n} \mathbf{S}_{n-1}
$$

De là on tire immédiatement:

$$
S_{n}=\frac{n}{a+n} \cdot \frac{n-1}{a+n-1} \cdot \frac{n-2}{a+n-2} \cdots \frac{1}{a+1} S_{0},
$$

et comme $\boldsymbol{S}_{0}=\frac{1}{a}$, on obtient l'expression

$$
\left.\frac{1.2 .3 \ldots n}{a(a+1)(a+2) \ldots(a+n)}=\frac{(n)_{0}}{a}-\frac{(n)_{1}}{a+1}+\frac{(n)_{2}}{a+2}-\frac{(n)_{3}}{a+3}+\cdots *\right)
$$

En fesant $a=-\frac{1}{x}$, on en tire

$$
\frac{(-1)^{n} 1.2 .3 \ldots n \cdot x^{n}}{(1-x)(1-2 x)(1-3 x) \ldots(1-n x)}=(n)_{0}-\frac{(n)_{1}}{1-x}+\frac{(n)_{2}}{1-2 x}-\cdots+\frac{(-1)^{n}}{1-n x} .
$$

Pour chasser le facteur $(-1)^{n}$, il n'y a qu'à renverser l'ordre des termes.

*) On parvient aussi à cette formule par l'intégrale

$$
\int_{0}^{1} x^{a-1}(1-x)^{n} d x=\frac{\Gamma(a) \Gamma(n+1)}{\Gamma(a+n+1)}=\frac{1.2 \ldots n}{a(a+1)(a+2) \ldots(a+n-1)} .
$$

Il n'y a qu'à développer le binôme $(1-x)^{n}$ et d'intégrer les termes de la série; mais nous préféront la démonstration élémentaire ci-dessus. 
Cela donne à l'aide de l'équation $(\boldsymbol{n})_{n-k}=(\boldsymbol{n})_{k}$ :

$$
\begin{aligned}
& 1.2 .3 \ldots n \cdot x^{n} \cdot \frac{1}{(1-x)(1-2 x) \ldots(1-n x)} \\
= & \frac{(n)_{0}}{1-n x}-\frac{(n)_{1}}{1-(n-1) x}+\frac{(n)_{2}}{1-(n-2) x}-\cdots
\end{aligned}
$$

Cette formule sert à déterminer $\bar{C}_{k}^{n}$; car en vertu de la formule (11.) de l'introduction, on a

$$
\begin{aligned}
& 1.2 \ldots n\left[\bar{C}_{0}^{n} x^{n}+\bar{C}_{1} x^{n+1}+\bar{C}_{2}^{n} x^{n+2}+\cdots\right] \\
= & \frac{(n)_{0}}{1-n x}-\frac{(n)_{1}}{1-(n-1) x}+\frac{(n)_{2}}{1-(n-2) x}-\cdots,
\end{aligned}
$$

et pour trouver $\overline{\boldsymbol{C}}_{k}$ il faut différentier $(\boldsymbol{n}+\boldsymbol{k})$ fois l'équation citée, et faire alors $x=0$; ou, ce qui revient au même, égaliser les coefficients de $x^{n+k}$ à gauche et à droite; ce qui donne

$$
\text { (3.) } \quad \bar{C}_{k}=\frac{(n)_{0} n^{n+k}-(n)_{1}(n-1)^{n+k}+(n)_{2}(n-2)^{n+k}-\cdots}{1.2 .3 \ldots n} \text {. }
$$

Cette équation fait voir que l'expression

$$
(n)_{0} n^{p}-(n)_{1}(n-1)^{p}+(n)_{2}(n-2)^{p}-\cdots,
$$

qui se trouve dans plusieurs résultats analytiques, représente le produit

$$
\text { (5.) } \quad 1.2 .3 \ldots n . \bar{C}_{p-n}^{n}
$$

et qu'elle se réduit à $1.2 .3 \ldots n$ pour $p=n$, et à zéro pour $p<n$. Les deux derniers circonstances sont connus; mais il paraît que la relation la plus générale qui existe entre les expressions ( 4 et 5.) n'a pas été reconnue.

\section{2.}

Du développement de $\left(\frac{x}{e^{x}-1}\right)^{n}$.

La méthode dont nous nous sommes servie pour trouver la valeur de $\bar{C}_{k}$ n'est pas également applicable à la détermination de $\stackrel{n}{C}_{k}$, parcequ'elle est fondée sur une décomposition de la fraction $1:(1-x)(1-2 x) \ldots(1-n x)$ en fractions simples, qui n'a pas lieu pour la faculté $z(z+1) \ldots(z+n-1)$. Pour déterminer les valeurs des coefficients $\stackrel{n}{C}_{0}, \stackrel{n}{C}_{1}$, etc., nous ferons voir d'abord que ces quantités entrent dans le développement de la fonction $\left(\frac{x}{e^{x}-1}\right)^{n}$, et que le problème se réduit à une diffẻrentiation répétée de cette fonction. 


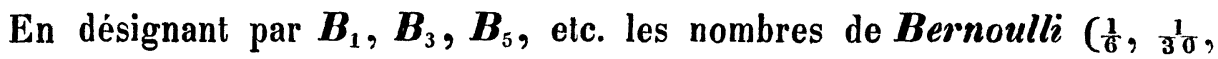
$\frac{1}{4^{2}}$, etc.), on a

$$
\frac{x}{e^{x}-1}=1-\frac{1}{2} x+\frac{B_{1} x^{2}}{1.2}-\frac{B_{3} x^{4}}{1.2 .3 .4}+\frac{B_{5} x^{6}}{1.2 .3 .4 .5 .6}-\cdots
$$

où la variable $x$ doit être comprise entre les limites $-2 \pi$ et $+2 \pi$. Cette équation est la même que celle dont s'est servi Laplace pour trouver immédiatement $\boldsymbol{B}_{k}$, savoir

$$
\left[\boldsymbol{D}^{k}\left(\frac{x}{e^{x}-1}\right)\right]_{(1)}=(-1)^{\frac{1}{2} k-1} \boldsymbol{B}_{k-1} \text { (où } k \text { est un nombre pair). }
$$

D'autre part il est clair qu'un résultat de la forme

$$
\text { (3.) }\left(\frac{x}{e^{x}-1}\right)^{n}=\stackrel{n}{A_{0}}-\stackrel{n}{A}_{1} x+\stackrel{n}{A}_{2} x^{2}-\stackrel{n}{A}_{3} x^{3}+\cdots
$$

peut être tiré de l'équation (1.) en désignant par $n$ un nombre entier positif et $\operatorname{par} \stackrel{n}{\boldsymbol{A}_{0}}, \stackrel{n}{\boldsymbol{A}_{1}}$, etc. des coefficients indéterminés.

Pour trouver une relation entre ces coefficients, nous prenons la dérivée de l'équation (3.) qui à gauche est

$$
n\left(\frac{x}{x^{x}-1}\right)^{n-1}\left[\frac{1}{e^{x}-1}-\frac{x e^{x}}{\left(e^{x}-1\right)^{2}}\right]=n \frac{x^{n-1}}{\left(e^{x}-1\right)^{n}}(1-x)-n \frac{x^{n}}{\left(e^{x}-1\right)^{n+1}} .
$$

Ayant multiplié cette derivée par $x$, on obtient

$n\left(\frac{x}{e^{x}-1}\right)^{n}(1-x)-n\left(\frac{x}{e^{x}-1}\right)^{n+1}=-1 \boldsymbol{A}_{1}^{n} x+2 \boldsymbol{A}_{2}^{n} x^{2}-3 \boldsymbol{A}_{3} x^{3}+\dot{4} \dot{A}_{4} x^{4}-\cdots$

Quant aux expressions à gauche, elles peuvent être développées à l'aide de l'équation (3.). En égalant après les coefficients de $x^{k}$ à gauche et à droite, on obtient sur le champ la relation

$$
\text { (4.) } \quad n \stackrel{n+1}{\boldsymbol{A}}_{k}=(\boldsymbol{n}-\boldsymbol{k}) \stackrel{n}{\boldsymbol{A}}_{k}+\boldsymbol{n} \ddot{\boldsymbol{A}}_{k-1} \text {. }
$$

Les coefficients qui satisfont à cette condition se divisent en deux classes, selon que $k<n$ ou $k \geqq n$. Dans le premier cas on peut se servir de la substitution

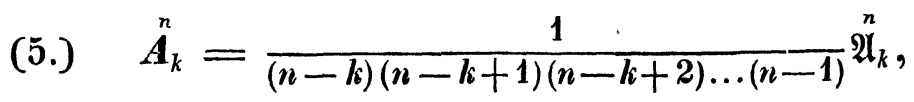

par laquelle la condition (4.) se change en

$$
\stackrel{n+1}{\mathfrak{A}_{k}}=\stackrel{n}{\mathfrak{A}_{k}}+\boldsymbol{n} \stackrel{n}{\mathfrak{A}}_{k-1} \text {. }
$$

Cette équation fait voir l'identité de $\stackrel{n}{\mathfrak{A}_{k}}$ et $\stackrel{n}{C_{k}}$, et delà on tire l'expression 
remarquable

$$
\begin{aligned}
& \left(\frac{x}{e^{x}-1}\right)^{n}=\stackrel{n}{C}_{0}-\frac{\stackrel{n}{C}_{1}}{n-1} x+\frac{\stackrel{n}{C}_{2}}{(n-1)(n-2)} x^{2}-\cdots+\frac{(-1)^{n-1} \stackrel{n}{n-1}_{(n-1)(n-2) \ldots 2.1}^{\prime}}{(n-1} \\
& +(-1)^{n}\left[\ddot{A}_{n} x^{n}-\hat{A}_{n+1} x^{n+1}+\stackrel{n}{A}_{n+2} x^{n+2}-\cdots\right] \text {. }
\end{aligned}
$$

Il suit de là que le coefficient $C_{k}$ peut être exprimé par

ou bien par

$$
\left[\boldsymbol{D}^{k}\left(\frac{x}{e^{x}-1}\right)^{n}\right]_{(0)}=\frac{(-1)^{k} \cdot 1.2 .3 \ldots k}{(n-1)(n-2) \ldots(n-k)} \stackrel{n}{C}_{k}
$$

$$
\text { (7.) } \quad \boldsymbol{C}_{k}^{n}=(-1)^{k}(\boldsymbol{n}-1)_{k} \cdot\left[\boldsymbol{D}^{k}\left(\frac{x}{e^{x}-1}\right)^{n}\right]_{(1)} \text {. }
$$

Cette formule offre, comme cas particulier, la détermination des nombres de Bernoulli, parcequ'en vertu de l'équation (2.) on a

$$
\text { (8.) } \quad \boldsymbol{B}_{k-1}=(-1)^{\frac{1}{k} k-1}\left[D^{k}\left(\frac{x}{e^{x}-1}\right)\right]_{(1)} \text {. }
$$

Il s'agit maintenant à développer les différentielles de la fonction $\left(\frac{x}{e^{x}-1}\right)^{n}$. Nous exécuterons cela par un procédé qui s'étend encore plus généralement sur le développement de $\left[D^{k}\left(\frac{1}{\varphi(x)}\right)^{n}\right]_{(0)}$, pourvu que $\varphi(x)$ soit une fonction qui se réduit à l'unité pour $x=0$.

\section{ऽ. 3.}

Développement de $\stackrel{n}{\boldsymbol{C}}_{k}$.

En désignant par $y$ la fonction

$$
\frac{e^{x}-1}{x}=1+\frac{1}{2} x+\frac{1}{6} x^{2}+\frac{1}{24} x^{3}+\cdots,
$$

on obtient à l'aide de la formule du binome:

$$
\begin{gathered}
\left(\frac{x}{e^{x}-1}\right)^{n}=\frac{1}{y^{n}}=\frac{1}{[1+(y-1)]^{n}} \\
=1+(-n)_{1}(y-1)+(-n)_{2}(y-1)^{2}+\cdots+(-n)_{k}(y-1)^{k} \\
+(-n)_{k+1}(y-1)^{k+1}+(-n)_{k+2}(y-1)^{k+2}+\cdots
\end{gathered}
$$

Cette série se divise en deux parties, dont la seconde se présente sous la forme

$$
a x^{k+1}+b x^{k+2}+c x^{k+3}+\cdots
$$

Substituant la valeur de $y-1$, la différentielle de l'ordre $k$ de celte partie s'évanouit pour $x=0$ et il reste 


$$
\begin{gathered}
\text { (1.) }\left[D^{k}\left(\frac{x}{e^{x}-1}\right)^{n}\right]_{(1)} \\
=\left[D^{k}\left\{1+(-n)_{1}(y-1)+(-n)_{2}(y-1)^{2}+\cdots+(-n)_{k}(y-1)^{k}\right\}\right]_{(())} .
\end{gathered}
$$

En développant les puissances de $y-1$, cette équation peut être transformée en celle ci :

(2.) $\left[D^{k}\left(\frac{x}{e^{x}-1}\right)^{n}\right]_{(I)}=\left[D^{k}\left(1+S_{1} y+S_{2} y^{2}+S_{3} y^{3}+\cdots+S_{k} y^{k}\right)\right]_{(1)}$ et on trouve sans peine que les coefficients $\boldsymbol{S}$ sont exprimés par

$$
\text { (3.) } \begin{aligned}
\quad S_{i}=( & (-n)_{i} i_{0}-(-n)_{i+1}(i+1)_{1}+(-n)_{i+2}(i+2)_{2}-\cdots \\
& \cdots(-1)^{k-i}(-n)_{k}(i+\overline{k-i})_{k-i} .
\end{aligned}
$$

Ayant égard aux relations connues

$$
\begin{array}{l|l}
(-n)_{i+1}=(-n)_{i} \frac{(-n)-i}{i+1} & (i+1)_{1}=\frac{i+1}{1} \\
-(-n)_{i+2}=(-n)_{i} \frac{(-n)-i}{i+1} \cdot \frac{(-n)-i-1}{i+2} & (i+2)_{2}=\frac{(i+1)(i+2)}{1.2}
\end{array}
$$

l'équation (3.) peut être remplacée par la suivante:

(4.) $S_{i}=(-n)_{i}\left[1+\frac{n+i}{1}+\frac{(n+i)(n+i+1)}{1.2}+\cdots+\frac{(n+i)(n+i+1) \ldots(n+k-1)}{1.2 .3 \ldots(k-i)}\right]$.

C'est ici où la formule sommatoire

$$
1+\frac{a}{1}+\frac{a(a+1)}{1.2}+\cdots+\frac{a(a+1) \ldots(a+q-1)}{1.2 \ldots q}=\frac{(a+1)(a+2)(a+3) \ldots(a+q)}{1.2 .3 \ldots q}
$$

peut être appliquée, et on en obtient

$$
S_{i}=(-n)_{i} \frac{(n+i+1)(n+i+2) \ldots(n+k)}{1.2 .3 \ldots(k-i)}
$$

ou ce qui revient au même:

$$
\boldsymbol{S}_{i}=(-1)^{i}(\boldsymbol{n}+\boldsymbol{i}-1)_{i}(\boldsymbol{n}+\boldsymbol{k})_{k-i} \text {. }
$$

Renversant l'ordre des termes de l'équation (2.), on obtient

$$
\begin{gathered}
{\left[D^{k}\left(\frac{x}{e^{x}-1}\right)^{n}\right]_{(1)}} \\
=(-1)^{k}\left[D^{k}\left\{(n+k)_{0}(n+k-1)_{k} y^{k}-(n+k)_{1}(n+k-2)_{k-1} y^{k-1}+\cdots\right\}\right]_{(0)} \cdot \\
\text { Posons, pour abréger, } \\
\text { (5.) }\left[D^{k} y^{h}\right]_{(x=1)}=Q_{h} .
\end{gathered}
$$

Alors l'équation précedente devient 


$$
\begin{aligned}
\text { (6.) } \quad & {\left[\boldsymbol{D}^{k}\left(\frac{\boldsymbol{x}}{\boldsymbol{e}^{x}-1}\right)^{n}\right]_{(\boldsymbol{l})} } \\
=(-1)^{k}\left[(\boldsymbol{n}+\boldsymbol{k})_{0}(\boldsymbol{n}+\boldsymbol{k}-1)_{k}\right. & \boldsymbol{Q}_{k}-(\boldsymbol{n}+\boldsymbol{k})_{1}(\boldsymbol{n}+\boldsymbol{k}-2)_{k-1} \boldsymbol{Q}_{k-1} \\
& \left.+(\boldsymbol{n}+\boldsymbol{k})_{2}(\boldsymbol{n}+\boldsymbol{k}-3)_{k-2} \boldsymbol{Q}_{k-2}-\cdots\right] .
\end{aligned}
$$

On trouve la valeur de $\boldsymbol{Q}_{h}$ simplement par la différentiation de l'équation

$$
x^{h} y^{h}=\left(e^{x}-1\right)^{h}=h_{0} e^{h x}-h_{1} e^{(h-1) x}+h_{2} e^{(h-2) x}-\cdots
$$

En appliquant la règle connue pour la différentiation des produits, on obtient

$$
\begin{aligned}
\boldsymbol{D}^{h+k}\left(\boldsymbol{x}^{h} \boldsymbol{y}^{h}\right)= & (\boldsymbol{h}+\boldsymbol{k})_{0} \boldsymbol{x}^{h}: \boldsymbol{D}^{h+k} y^{h}+(\boldsymbol{h}+\boldsymbol{k})_{\mathbf{1}} \boldsymbol{h} \boldsymbol{x}^{h-1} \cdot \boldsymbol{D}^{h+k-1} \boldsymbol{y}^{h}+\cdots \\
& \cdots+(\boldsymbol{h}+\boldsymbol{k})_{h} \boldsymbol{h}(\boldsymbol{h}-\mathbf{1})(\boldsymbol{h}-2) \ldots \mathbf{2} \boldsymbol{1} \cdot \boldsymbol{D}^{k} y^{h} \\
= & (\boldsymbol{h})_{0} \boldsymbol{h}^{h+k} \boldsymbol{e}^{h x}-(\boldsymbol{h})_{\mathbf{1}}(\boldsymbol{h}-\mathbf{1})^{h+k} \boldsymbol{e}^{(h-1) x}+(\boldsymbol{h})_{2}(\boldsymbol{h}-\mathbf{2})^{h+k} \boldsymbol{e}^{(h-2) x}-\cdots
\end{aligned}
$$

Cette équation donne pour $x=0$ :

$$
\begin{aligned}
& (h+k)_{h} h(h-1)(h-2) \ldots 2.1\left[D^{k} y^{h}\right]_{(())} \\
& =(\boldsymbol{h})_{0} \boldsymbol{h}^{h+k}-(\boldsymbol{h})_{1}(\boldsymbol{h}-\mathbf{1})^{h+k}+(\boldsymbol{h})_{2}(\boldsymbol{h}-\boldsymbol{2})^{h+k}-\cdots \text {, }
\end{aligned}
$$

ce qui est le même que

$$
(h+k)(h+k-1)(h+k-2) \ldots(k-1) \cdot Q_{h}=1.2 \ldots h \cdot \bar{C}_{k}^{h} .
$$

De là on tire

et en vertu de l'équation (6.):

$$
\boldsymbol{Q}_{h}=\frac{1}{(h+k)_{h}} \overline{\boldsymbol{C}}_{k}^{h}
$$

$$
\begin{gathered}
\text { (7.) } \quad(-1)^{k}\left[D^{k}\left(\frac{x}{e^{x}-1}\right)^{n}\right]_{(1)} \\
=\frac{(n+k)_{0}(n+k-1)_{k}}{(2 k)_{k}} \bar{C}_{k}^{k}-\frac{(n+k)_{1}(n+k-2)_{k-1}-(k-1)}{(2 k-1)_{k-1}} C_{k} \\
\quad+\frac{(n+k)_{2}(n+k-3)_{k-2}-\left(k_{k-2)}\right.}{(2 k-2)_{k-2}}-\cdots
\end{gathered}
$$

Cette formule donne tous les coefficients du développement de $\left(\frac{x}{e^{x}-1}\right)^{n}$. En se bornant au cas $k<n$, on a en vertu de (7. \$. 2.):

$$
\begin{aligned}
& \stackrel{n}{C}_{k}=(n-1)_{k}\left[\frac{(n+k)_{0}(n+k-1)_{k}}{(2 k)_{k}} \bar{C}_{k}^{k}-\frac{(n+k)_{1}(n+k-2)_{k-1}}{(2 k-1)_{k-1}}-\stackrel{(k-1)}{C_{k}}\right. \\
& \left.+\frac{(n+k)_{2}(n+k-3)_{k-2}}{(2 k-2)_{k-2}} \stackrel{(k-2)}{C_{k}}-\cdots\right] .
\end{aligned}
$$

Il $y$ a encore à observer que le cas $n=1$ fournit une formule pour les nombres de Bernoulli, car à l'aide de la formule (8. \$. 2.) on obtient

$$
\begin{aligned}
\boldsymbol{B}_{k-1}=(-1)^{\frac{1}{2} k-1}\left[\frac{(k+1)_{0}}{(2 k)_{k}} \bar{C}_{k}^{-k}\right. & -\frac{(k+1)_{1}}{(2 k-1)_{k-1}} \stackrel{(k-1)}{C}_{k}^{-(k-2)} \\
& \left.+\frac{(k+1)_{2}}{(2 k-2)_{k-2}} \stackrel{C}{K}^{-2}-\cdots\right]
\end{aligned}
$$


$k$ élant un nombre pair $>1$. Si $k$ est impair et $>1$, la quantité entre les crochets s'évanouit, parceque le développement de $\frac{x}{e^{x}-1}$ ne contient pas des puissances impaires de $x$ d'un exposant $>1$.

\section{S. 4.}

Développement de $[l(1+x)]^{m}$.

La formule du binôme peut être transformée aisément à l'aide de la relation

$u(u-1)(u-2) \ldots(u-(n-\dot{1}))=\ddot{C}_{0} u^{n}-\stackrel{n}{C}_{1} u^{n-1}+\stackrel{n}{C}_{2} u^{n-2}-\cdots+(-1)^{n-1} \stackrel{n}{C}_{n-1} u$ et on trouve par là, sous condition que $1>x>-1$ :

$$
\begin{aligned}
(1+x)^{u}=1 & +\frac{u}{1} x+\frac{u(u-1)}{1.2} x^{2}+\ldots \\
=1 & +\left(\grave{C}_{1} x-\frac{1}{2} \grave{C}_{1}^{2} x^{2}+\frac{1}{2.3} \grave{C}_{2}^{3} x^{3}-\ldots\right) \frac{u}{1} \\
& +\left(\grave{C}_{0}^{2} x^{2}-\frac{1}{3} \grave{C}_{2}^{3} x^{3}+\frac{1}{3.4} \grave{C}_{3} x^{4}-\ldots\right) \frac{u^{2}}{1.2} \\
& +\ldots \ldots
\end{aligned}
$$

Comme d'autre part on a

$$
(1+x)^{u}=1+l(1+x) \frac{u}{1}+[l(1+x)]^{2} \frac{u^{2}}{1.2}+\cdots,
$$

les coefficients des mêmes puissances de $u$ peuvent être égalisés. Cela donne

$$
\text { (1.) } \begin{aligned}
{[l(1+x)]^{m}=\stackrel{m}{C}_{0} x^{m} } & -\frac{m+1}{m+1} x^{m+1}+\frac{\stackrel{m}{C}_{2}}{(m+1)(m+2)} x^{m+2}-\cdots \\
1 & >x>-1 .
\end{aligned}
$$

Cette formule peut servir à transformer une série de la forme

$$
a l(1+x)+b[l(1+x)]^{2}+c[l(1+x)]^{2}+\cdots
$$

en une autre qui ne contient que des puissances de $x$. On a par exemple

$$
\begin{gathered}
\frac{1}{1+l(1+x)}=1-l(1+x)+[l(1+x)]^{2}-\cdots \\
1>l(1+x)>-1
\end{gathered}
$$

et par conséquent

$$
\begin{aligned}
& \text { (2.) } \frac{1}{1+l(1+x)}=1-A_{1} x+A_{2} x^{2}-A_{3} x^{3}+\cdots \\
& 1>l(1+x)>-1, \quad 1>x>-1 \text {. }
\end{aligned}
$$


Le coefficient $\boldsymbol{A}_{k}$ peut être trouvé par la formule

$$
\boldsymbol{A}_{k}=\stackrel{k}{\boldsymbol{C}}_{0}+\frac{1}{k} \stackrel{k}{\boldsymbol{C}_{1}}+\frac{1}{k(k-1)} \stackrel{k}{C}_{2}+\cdots+\frac{1}{k \ldots 2} \stackrel{k}{\boldsymbol{C}}_{k-1} \text {. }
$$

Les deux conditions énoncées pour l'équation (2.) peuvent être réduites à une seule, savoir à $1>x>\frac{1}{e}-1$.

Passons à une autre application de la formule (1.). De l'intégrale

$$
\int_{0}^{1}\left(l \frac{1}{z}\right)^{n-1} z^{a-1} d z=\frac{\Gamma(n)}{a^{n}}=\frac{1.2 \ldots(n-1)}{a^{n}}
$$

on tire $z=1-x$ :

$$
\int_{0}^{1}[-l(1-x)]^{n-1}(1-x)^{a-1} d x=\frac{1.2 \ldots(n-1)}{a^{n}} .
$$

Maintenant la fonction $[-l(1-x)]^{n-1}$ peut être développée. En intégrant les termes de la série, on obtient

$$
\frac{1}{a^{n}}=\frac{1}{a(a+1) \ldots(a+n-1)}+\frac{\stackrel{n}{C}_{1}}{a(a+1) \ldots(a+n)}+\frac{\stackrel{n+1}{C}_{2}}{a(a+1) \ldots(a+n+1)}+\cdots
$$

Ce résultat, dont ont fait usage dans le calcul inverse des différences, a élé déjà donné par Stirling.

S. 5.

Développement de $D^{n} f(l x)$.

La différentiation réiterée de la fonction. $f(l x)$ donne les expressions

$$
\begin{aligned}
& D f(l x)=\frac{1}{x} f^{\prime}(l x), \\
& D^{2} f(l x)=\frac{1}{x^{2}}\left[f^{\prime \prime}(l x)-f^{\prime}(l x)\right] \\
& D^{3} f(l x)=\frac{1}{x^{3}}\left[f^{\prime \prime \prime}(l x)-3 f^{\prime \prime}(l x)+2 f^{\prime}(l x)\right]
\end{aligned}
$$

On en conclut que l'expression générale est de la forme

$$
D^{n} f(l x)=\frac{1}{x^{n}}\left[\ddot{A}_{0} f^{(n)}(l x)-\ddot{A}_{1} f^{(n-1)}(l x)+\hat{A}_{2} f^{(n-2)}(l x)-\cdots\right] .
$$

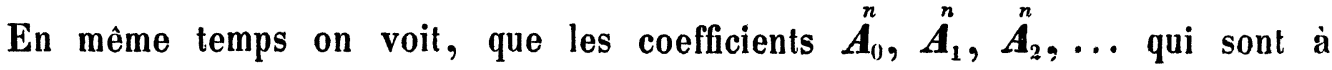
déterminer, ne dependent pas de la nature de la fonction $f$. Leurs valeurs peuvent dont être trouvées si l'on prend une fonction $f$ telle, que les différentiations à droite et à gauche dans l'équation ci-dessus sont praticables. 
La plus simple fonction de cette espèce est $f(y)=e^{-\beta y}$, et on en obtient

$$
f^{(k)}(y)=(-1)^{k} \beta^{k} e^{-\beta y}, \quad f^{(k)}(l x)=(-1)^{k} \beta^{k} x^{-k},
$$

parcequ'on a immédiatement

$$
f(l x)=x^{-\beta} \quad \text { et } \quad D^{n} f(l x)=(-1)^{n} \beta(\beta+1)(\beta+2) \ldots(\beta+n-1) x^{-\beta-n} .
$$

La formule (1.) donne maintenant en substituant les valeurs mentionnées:

$$
\boldsymbol{\beta}(\boldsymbol{\beta}+1)(\boldsymbol{\beta}+2) \ldots(\boldsymbol{\beta}+\boldsymbol{n}-1)=\stackrel{n}{\boldsymbol{A}_{0}} \boldsymbol{\beta}^{n}+\stackrel{n}{\boldsymbol{A}}_{1} \boldsymbol{\beta}^{n-1}+\stackrel{n}{\boldsymbol{A}}_{2} \boldsymbol{\beta}^{n-2}+\cdots+\boldsymbol{A}_{n-1} \boldsymbol{\beta} .
$$

Cette équation fait voir que les coefficients $\stackrel{n}{A}_{0}, \ddot{A}_{1}, \mathscr{A}_{2}, \ldots$ sont ceux de la faculté $\left(\beta_{0}+1\right)^{n}$; donc on obtient la formule remarquable suivante:

(2.) $\quad D^{n} f(l x)=\frac{1}{x^{n}}\left[\stackrel{n}{C_{0}} f^{(n)}(l x)-\stackrel{n}{C_{1}} f^{(n-1)}(l x)+\stackrel{n}{C_{2}} f^{(n-2)}(l x)-\cdots\right]$

Pour faciliter les calculs numériques, nous ajoutons une petite table des coefficients $\stackrel{n}{\boldsymbol{C}}_{k}$ et $\overline{\boldsymbol{C}}_{k}^{n}$.

$\begin{array}{lrrrrrrrrrrrr}n= & - \text { IV } & - \text { III } & - \text { II } & -1 & + \text { I } & + \text { II } & + \text { III } & + \text { IV } & + \text { V } & + \text { VI } & + \text { VII } & + \text { VIII } \\ \boldsymbol{C}_{0}= & 1 & 1 & 1 & 1 & 1 & 1 & 1 & 1 & 1 & 1 & 1 & 1 \\ C_{1}= & 10 & 6 & 3 & 1 & & 1 & 3 & 6 & 10 & 15 & 21 & 28 \\ C_{2}= & 65 & 25 & 7 & 1 & & 2 & 11 & 35 & 85 & 175 & 322 \\ C_{3}= & 350 & 90 & 15 & 1 & & & 6 & 50 & 225 & 735 & 1960 \\ C_{4}= & 1701 & 301 & 31 & 1 & & & & 24 & 274 & 1624 & 6769 \\ C_{5}= & 7770 & 966 & 63 & 1 & & & & & 120 & 1764 & 13132 \\ C_{6}= & 35105 & 3025 & 127 & 1 & & & & & & 720 & 13068 \\ C_{7}= & 149750 & 9330 & 255 & 1 & & & & & & & 5040\end{array}$

Dresde, Décembre 1851. 